\title{
Global Existence and Stability for Neutral Functional Evolution Equations with State-Dependent Delay
}

Abstract: In this paper we prove the global existence and attractivity of mild solutions for neutral semilinear evolution equations with state-dependent delay in a Banach space.

Keywords: Semilinear functional differential equations, mild solution, attractivity, evolution system, fixedpoint, infinite delay, infinite interval

AMS: $34 \mathrm{G} 20,34 \mathrm{~K} 20,34 \mathrm{~K} 30$

DOI 10.2478/msds-2014-0006

Received November 22, 2013; accepted May 12, 2014.

\section{Introduction}

In this paper, we consider the existence and attractivity of mild solutions of the following neutral evolution equation with state- dependent delay

$$
\begin{cases}\frac{d}{d t}\left[y(t)-g\left(t, y_{\rho\left(t, y_{t}\right)}\right)\right]-A(t) y(t)=f\left(t, y_{\rho\left(t, y_{t}\right)}\right), & t \in J:=[0, \infty) \\ y(t)=\phi(t) & t \in(-\infty, 0],\end{cases}
$$

where $\mathcal{B}$ is an abstract phase space to be specified later, $f$ and $g$ are given functions from $J \times \mathcal{B}$ into $E$, the function $\rho: J \times \mathcal{B} \rightarrow \mathbb{R}$ is an appropriate function, $\phi \in \mathcal{B}$ and $\{A(t)\}_{0 \leq t<+\infty}$ is a family of linear closed (not necessarily bounded) operators from $E$ into $E$ that generate an evolution system of operators $\{U(t, s)\}_{(t, s) \in J \times J}$ for $0 \leq s \leq t<+\infty$.

For any continuous function $y$ and any $t \geq 0$, we denote by $y_{t}$ the element of $\mathcal{B}$ defined by $y_{t}(\theta)=y(t+\theta)$ for $\theta \in(-\infty, 0]$. Here $y_{t}(\cdot)$ represents the history of the state up to the present time $t$. We assume that the histories $y_{t}$ belong to $\mathcal{B}$.

In many fields of science we can describe various evolutionary process by differential equations with delay and for this reason the study of this type of equations has received great attention during the last years. The literature on differential equations with delay is very extensive, we refer, for instance to the books by Hale [13], Hale and Verduyn Lunel [15], Lakshmikantham et al. [20], Kolmanovskii and Myshkis [19] and Wu [23]. Some recent results on various classes of semilinear functional differential equations are reported in [6, 22]. In this paper we are interested in the existence and attractivity of mild solutions of neutral state-dependent delay functional differential equations. In the literature there are many papers studying these equations using

Abdessalam Baliki: Laboratory of Mathematics, University of Sidi Bel-Abbes, PO Box 89, 22000 Sidi Bel-Abbes, Algeria, E-mail: adsbaliki@yahoo.fr

*Corresponding Author: Mouffak Benchohra: Laboratory of Mathematics, University of Sidi Bel-Abbes, PO Box 89, 22000 Sidi Bel-Abbes, Algeria

and Department of Mathematics, Faculty of Science, King Abdulaziz University, P.O. Box 80203, Jeddah 21589, Saudi Arabia, E-mail: benchohra@univ-sba.dz 
different methods. Using the fixed point method combined with the semigroup theory in Fréchet space, Baghli and Benchohra [3-5] considered some classes of neutral evolution equations on unbounded intervals. On other hand, for the local existence, we refer, for instance, to the papers by Aiello et al. [2], dos Santos [10], Hernández et al. [16, 17], and the references therein.

In this paper, sufficient conditions are provided to get the global existence and attractivity of mild solutions of the problem (1) using Burton-Kirk's fixed point theorem (see [7]). Let us mention that under a Lipschitz condition on the nonlinearities, one can get a uniqueness result. As far as we know, there are few papers dealing with global existence results for the problem (1). Most of these results are stated in the Fréchet space setting. The present paper provides sufficient conditions for the existence and attractivity mild solutions to problem (1) in the Banach space setting.

\section{Preliminary}

Let $E$ a Banach space with norm $|\cdot|$ and $B C(J, E)$ the Banach space of all bounded and continuous functions $y$ mapping $J$ into $E$ with the usual supremum norm

$$
\|y\|=\sup _{t \in J}|y(t)| .
$$

Let $X$ is the space defined by

$$
X=\left\{y: \mathbb{R} \rightarrow E \text { such that }\left.y\right|_{J} \in B C(J, E) \text { and } y_{0} \in \mathcal{B}\right\} .
$$

We denote by $\left.y\right|_{J}$ the restriction of $y$ to $J$.

In this paper, we will employ an axiomatic definition of the phase space $\mathcal{B}$ introduced by Hale and Kato in [14] and follow the terminology used in [18]. Thus, $\left(\mathcal{B},\|\cdot\|_{\mathcal{B}}\right)$ will be a seminormed linear space of functions mapping $(-\infty, 0]$ into $E$, and satisfying the following axioms :

$\left(A_{1}\right)$ If $y:(-\infty, b) \rightarrow E, b>0$, is continuous on $[0, b]$ and $y_{0} \in \mathcal{B}$, then for every $t \in[0, b)$ the following conditions hold :

(i) $y_{t} \in \mathcal{B}$;

(ii) There exists a positive constant $H$ such that $|y(t)| \leq H\left\|y_{t}\right\|_{\mathcal{B}}$;

(iii) There exist two functions $K(\cdot), M(\cdot): \mathbb{R}_{+} \rightarrow \mathbb{R}_{+}$independent of $y$ with $K$ continuous and $M$ locally bounded such that:

$$
\left\|y_{t}\right\|_{\mathcal{B}} \leq K(t) \sup \{|y(s)|: 0 \leq s \leq t\}+M(t)\left\|y_{0}\right\|_{\mathcal{B}} .
$$

$\left(A_{2}\right)$ For the function $y$ in $\left(A_{1}\right), y_{t}$ is a $\mathcal{B}$-valued continuous function on $[0, b]$.

$\left(A_{3}\right)$ The space $\mathcal{B}$ is complete.

Remark 2.1. In the sequel we assume that $K$ and $M$ are bounded on $J$ and

$$
y:=\max \left\{\sup _{t \in \mathbb{R}_{+}}\{K(t)\}, \sup _{t \in \mathbb{R}_{+}}\{M(t)\}\right\} .
$$

For other details we refer, for instance to the book by Hino et al [18].

In what follows, we assume that $\{A(t), t \geq 0\}$ is a family of closed densely defined linear unbounded operators on the Banach space $E$ and with domain $D(A(t))$ independent of $t$.

Definition 2.2. A family of bounded linear operators

$$
\{U(t, s)\}_{(t, s) \in \Delta}: U(t, s): E \rightarrow E(t, s) \in \Delta:=\{(t, s) \in J \times J: 0 \leq s \leq t<+\infty\}
$$

is called en evolution system if the following properties are satisfied: 
1. $U(t, t)=I$ where $I$ is the identity operator in $E$,

2. $U(t, s) U(s, \tau)=U(t, \tau)$ for $0 \leq \tau \leq s \leq t<+\infty$,

3. $U(t, s) \in B(E)$ the space of bounded linear operators on $E$, where for every $(s, t) \in \Delta$ and for each $y \in E$, the mapping $(t, s) \rightarrow U(t, s) y$ is continuous.

More details on evolution systems and their properties could be found on the books of Ahmed [1], Engel and Nagel [11] and Pazy [21].

Lemma 2.3. [8][Corduneanu]

Let $C \subset B C(J, E)$ be a set satisfying the following conditions:

(i) $C$ is bounded in $B C(J, E)$;

(ii) the functions belonging to $C$ are equicontinuous on any compact interval of J;

(iii) the set $C(t):=\{y(t): y \in C\}$ is relatively compact on any compact interval of $J$;

(iv) the functions from $C$ are equiconvergent, i.e., given $\varepsilon>0$, there corresponds $T(\varepsilon)>0$ such that $\mid y(t)-$ $y(+\infty) \mid<\varepsilon$ for any $t \geq T(\varepsilon)$ and $y \in C$.

Then $C$ is relatively compact in $B C(J, E)$.

Theorem 2.4. [7][Burton-Kirk's fixed point theorem]

Let $X$ Banach space, and $A, B: X \rightarrow X$ two operators. Suppose that $B$ is a contraction and A a compact operator. Then either

(i) $x=\lambda B\left(\frac{x}{\lambda}\right)+\lambda A x$ has a solution for $\lambda=1$, or

(ii) the set $\left\{x \in X: x=\lambda B\left(\frac{x}{\lambda}\right)+\lambda A x, \lambda \in(0,1)\right\}$ is unbounded.

\section{Main result}

Definition 3.1. A function $y \in X$ is said to be a mild solution of the problem (1), if

$$
y(t)= \begin{cases}\phi(t), & \text { if } t \leq 0 \\ U(t, 0)(\phi(0)-g(0, \phi))+g\left(t, y_{\rho\left(t, y_{t}\right)}\right) & \\ +\int_{0}^{t} U(t, s) A(s) g\left(s, y_{\rho\left(s, y_{s}\right)}\right) d s+\int_{0}^{t} U(t, s) f\left(s, y_{\rho\left(s, y_{s}\right)}\right) d s, & \text { if } t \in J .\end{cases}
$$

To prove our results we introduce the following conditions:

$\left(H_{1}\right)$ There exists a constant $\widehat{M} \geq 1$ and $\omega>0$ such that

$$
\|U(t, s)\|_{B(E)} \leq \widehat{M} e^{-\omega(t-s)} \text { for every }(s, t) \in \Delta .
$$

$\left(H_{2}\right)$ There exists a function $p \in L^{1}\left(J, \mathbb{R}_{+}\right)$such that:

$$
|f(t, u)| \leq p(t)\left(\|u\|_{\mathcal{B}}+1\right) \text { for a.e. } t \in J \text { and each } u \in \mathcal{B} .
$$

$\left(H_{3}\right)$ For each $(t, s) \in \Delta$ we have

$$
\lim _{t \rightarrow+\infty} \int_{0}^{t} e^{-w(t-s)} p(s) d s=0 .
$$


$\left(H_{4}\right)$ There exists a constant $\widetilde{M}>0$ such that:

$$
\left\|A^{-1}(t)\right\|_{B(E)} \leq \widetilde{M} \text { for all } t \in J .
$$

$\left(H_{5}\right)$ There exists a constant $\ell>0$ such that

$$
|A(t) g(t, \phi)-A(s) g(s, \varphi)| \leq \ell\left(|t-s|+\|\phi-\varphi\|_{\mathcal{B}}\right)
$$

for all $t, s \in J$ and $\phi, \varphi \in \mathcal{B}$.

$\left(H_{6}\right)$ There exists a bounded continuous function $\zeta: J \rightarrow \mathbb{R}_{+}$such that:

$$
|A(t) g(t, \phi)| \leq \zeta(t)\|\phi\|_{\mathcal{B}} \text { for all } t \in J, \phi \in \mathcal{B} .
$$

$\left(H_{7}\right)$ Let $\mathcal{R}_{\rho}^{-}=\left\{\rho\left(t, y_{t}\right): \rho\left(t, y_{t}\right) \leq 0,\left(t, y_{t}\right) \in \mathcal{B}\right\}$, and $\phi_{t}: \mathcal{R}_{\rho}^{-} \rightarrow \mathcal{B}$ continuous function and there exists a continuous and bounded function $L^{\phi}: \mathcal{R}_{\rho}^{-} \rightarrow(0, \infty)$ such that $\left\|\phi_{t}\right\| \leq L^{\phi}(t)\|\phi\|_{\mathcal{B}}$ for all $t \in \mathcal{R}_{\rho}^{-}$.

Remark 3.2. For more detailed information concerning $\left(H_{7}\right)$ see ([18], Lemma 3.4 and Proposition 7.1.1).

For the proof of our results, we are going to need the following lemma.

Lemma 3.3 ([16], Lemma 3.1). Let $y:(-\infty, \infty) \rightarrow E$ continuous and bounded and $y_{0}=\phi$. If $\left(H_{7}\right)$ holds, then

$$
\left\|y_{s}\right\|_{\mathcal{B}} \leq\left(M_{b}+L^{\phi}\right)\|\phi\|_{\mathcal{B}}+K_{b} \sup \{|y(\theta)| ; \theta \in[0, \max \{0, s\}]\}, s \in \mathcal{R}\left(\rho^{-}\right) \cup J,
$$

where $L^{\phi}=\sup _{t \in \mathcal{R}\left(\rho^{-}\right)} L^{\phi}(t)$.

Theorem 3.4. If assumptions $\left(H_{1}\right)-\left(H_{7}\right)$ are satisfied, and if

$$
y\left(\ell \widetilde{M}+\frac{\widehat{M}}{\omega}\right)<1
$$

and

$$
\widetilde{M} \zeta^{\star} y+\frac{\widehat{M} \zeta^{\star}}{\omega}+\widehat{M} y<1,
$$

then the problem (1) admits at least one mild solution.

Proof. It is clear that we will obtain the results if we show that the operator $T: X \rightarrow X$ defined by:

$$
T y(t)= \begin{cases}\phi(t), & \text { if } t \leq 0 \\ U(t, 0)(\phi(0)-g(0, \phi))+g\left(t, y_{\rho\left(t, y_{t}\right)}\right) & \\ +\int_{0}^{t} U(t, s) A(s) g\left(s, y_{\rho\left(s, y_{s}\right)}\right) d s+\int_{0}^{t} U(t, s) f\left(s, y_{\rho\left(s, y_{s}\right)}\right) d s, & \text { if } t \in J,\end{cases}
$$

has a fixed point.

For $\phi \in \mathcal{B}$, Let $x:(-\infty,+\infty) \rightarrow E$ be the function defined by

$$
x(t)= \begin{cases}\phi(t), & \text { if } t \in(-\infty, 0] \\ U(t, 0) \phi(0) & \text { if } t \in J .\end{cases}
$$

Then $x_{0}=\phi$. For each function $z \in \mathcal{X}$, we denote

$$
y(t)=x(t)+z(t) .
$$


It is obvious that $y$ satisfies (3) if and only if $z$ satisfies $z_{0}=0$ and for all $t \in J$

$$
\begin{aligned}
z(t)= & U(t, 0) g(0, \phi)+g\left(t, x_{\rho\left(s, x_{s}+z_{s}\right)}+z_{\rho\left(s, x_{s}+z_{s}\right)}\right)+\int_{0}^{t} U(t, s) A(s) g\left(s, x_{\rho\left(s, z_{s}+x_{s}\right)}+z_{\rho\left(s, z_{s}+x_{s}\right)}\right) d s \\
& +\int_{0}^{t} U(t, s) f\left(t, x_{\rho\left(s, x_{s}+z_{s}\right)}+z_{\rho\left(s, x_{s}+z_{s}\right)}\right) d s .
\end{aligned}
$$

In the sequel, we always denote $X_{0}$ is Banach space defined by

$$
X_{0}=\left\{z \in X: z_{0}=0\right\},
$$

endowed with the norm

$$
\|z\| x_{0}=\sup _{t \in J}|z(t)|+\left\|z_{0}\right\|_{\mathcal{B}}=\sup _{t \in J}|z(t)| .
$$

Now, we can decompose (4) as $z(t)=F z(t)+L z(t)$ which $F, L: X_{0} \rightarrow X_{0}$ given by

$$
F z(t)=\int_{0}^{t} U(t, s) f\left(s, x_{\rho\left(s, z_{s}+x_{s}\right)}+z_{\rho\left(s, z_{s}+x_{s}\right)}\right) d s, \text { for } t \in J,
$$

and

$$
L z(t)=U(t, 0) g(0, \phi)+g\left(t, x_{\rho\left(s, z_{s}+x_{s}\right)}+z_{\rho\left(s, z_{s}+x_{s}\right)}\right)+\int_{0}^{t} U(t, s) A(s) g\left(s, x_{\rho\left(s, z_{s}+x_{s}\right)}+z_{\rho\left(s, z_{s}+x_{s}\right.}\right) d s .
$$

Obviously the problem (1) has a solution is equivalent to $F+L$ has a fixed point. To prove this end, we start with the following estimate.

For each $z \in X_{0}$ and $t \in J \cup \mathcal{R}\left(\rho^{-}\right)$, we have

$$
\begin{aligned}
\left\|z_{t}+x_{t}\right\|_{\mathcal{B}} & \leq\left\|z_{t}\right\|_{\mathcal{B}}+\left\|x_{t}\right\|_{\mathcal{B}} \\
& \leq K(t)|z(t)|+K(t)\|U(t, 0)\|_{B(E)}\|\phi\|_{\mathcal{B}}+\left(M(t)+L^{\phi}\right)\|\phi\|_{\mathcal{B}} \\
& \leq y\|z\|_{x_{0}}+y \widehat{M} e^{-\omega t}\|\phi\|_{\mathcal{B}}+\left(y+L^{\phi}\right)\|\phi\|_{\mathcal{B}} \\
& \leq y\|z\|_{x_{0}}+\left[y(\widehat{M}+1)+L^{\phi}\right]\|\phi\|_{\mathcal{B}} .
\end{aligned}
$$

Now, we will show that the operators $F, L$ satisfy the conditions of Theorem 2.4.

\section{Step 1.}

$F$ is continuous and compact.

- $F$ is continuous. Let $\left(z^{k}\right)_{k \in \mathbb{N}}$ be a sequence in $X_{0}$ such that $z^{k} \rightarrow z$ in $X_{0}$, then For every $t \in J$ we obtain

$$
\begin{aligned}
\left|F\left(z^{k}\right)(t)-F(z)(t)\right| & \leq \int_{0}^{t}\|U(t, s)\|_{B(E)}\left|f\left(t, x_{\rho\left(t, x_{s}+z_{s}^{k}\right)}+z_{\rho\left(t, x_{s}+z_{s}^{k}\right)}^{k}\right)-f\left(t, x_{\rho\left(s, x_{s}+z_{s}\right)}+z_{\rho\left(s, x_{s}+z_{s}\right)}\right)\right| d s \\
& \leq \widehat{M} \int_{0}^{t} e^{-\omega(t-s)}\left|f\left(s, z_{\rho\left(t, x_{s}+z_{s}^{k}\right)}^{k}+x_{\rho\left(t, x_{s}+z_{s}^{k}\right)}\right)-f\left(s, z_{\rho\left(s, x_{s}+z_{s}\right)}+x_{\rho\left(s, x_{s}+z_{s}\right)}\right)\right| d s .
\end{aligned}
$$

Hance, from the continuity of the function $f$ and the Lebesgue dominated convergence theorem we obtain

$$
\left\|F z_{k}-F z\right\|_{x_{0}} \rightarrow 0 \text { as } k \rightarrow+\infty .
$$

So $F$ is continuous. 
- $F(D)$ relatively compact.

Let $D$ be a bounded subset of $X_{0}$. To show that $F(D)$ is relatively compact we will use Lemma 2.3.

Let $\eta \geq 0$ such that $D=\left\{z \in X_{0}:\|x\| x_{0} \leq \eta\right\}$. Let $z \in D$, then

$$
\begin{aligned}
|F(z)(t)| & \leq \int_{0}^{t}\|U(t, s)\|_{B(E)}\left|f\left(s, z_{\rho\left(s, x_{s}+z_{s}\right)}+x_{\rho\left(s, x_{s}+z_{s}\right)}\right)\right| d s \\
& \left.\leq \widehat{M} \int_{0}^{t} e^{-\omega(t-s)} p(s)\left(\| z_{\rho\left(s, x_{s}+z_{s}\right)}+x_{\rho\left(s, x_{s}+z_{s}\right.}\right) \|_{\mathcal{B}}+1\right) d s . \\
& \leq \widehat{M}\left(y\|z\|_{x_{0}}+\left(y(\widehat{M}+1)+L^{\phi}\right)\|\phi\|_{\mathcal{B}}+1\right) \int_{0}^{t} e^{-\omega(t-s)} p(s) d s \\
& \leq \widehat{M} \xi\|p\|_{L^{1}},
\end{aligned}
$$

with

$$
\xi:=y \eta+\left(y(\widehat{M}+1)+L^{\phi}\right)\|\phi\|_{\mathcal{B}}+1
$$

Thus $F(D)$ is bounded.

- $F(D)$ is equicontinuous.

Let $s, t \in[0, b]$ with $t>s$ and $z \in D$. Then, we have

$$
\begin{aligned}
|(F z)(t)-(F z)(s)|= & \mid \int_{0}^{s}(U(t, \tau)-U(s, \tau)) f\left(\tau, z_{\rho\left(\tau, x_{\tau}+z_{\tau}\right)}+x_{\rho\left(\tau, x_{\tau}+z_{\tau}\right)}\right) d \tau \\
& +\int_{s}^{t} U(t, \tau) f\left(\tau, z_{\rho\left(\tau, x_{\tau}+z_{\tau}\right)}+x_{\rho\left(\tau, x_{\tau}+z_{\tau}\right)}\right) d \tau \mid \\
\leq & \int_{0}^{s}\|U(t, \tau)-U(s, \tau)\|_{B(E)} p(\tau)\left(\left\|z_{\rho\left(\tau, x_{\tau}+z_{\tau}\right)}+x_{\rho\left(\tau, x_{\tau}+z_{\tau}\right)}\right\|_{\mathcal{B}}+1\right) d \tau \\
& +\widehat{M} \int_{s}^{t} e^{-\omega(t-\tau)} p(\tau)\left(\left\|z_{\rho\left(\tau, x_{\tau}+z_{\tau}\right)}+x_{\rho\left(\tau, x_{\tau}+z_{\tau}\right)}\right\|_{\mathcal{B}}+1\right) d \tau .
\end{aligned}
$$

Now, by the inequality (5) we get

$$
|(F z)(t)-(F z)(s)| \leq \xi \int_{0}^{s}\|U(t, \tau)-U(s, \tau)\|_{B(E)} p(\tau) d \tau+\widehat{M} \xi \int_{s}^{t} p(\tau) d \tau .
$$

The right-hand side of the above inequality tends to zero as $t-s \rightarrow 0$, which implies that $F(D)$ is equicontinuous.

Now, we will prove that $\Lambda:=\{(F z)(t): z \in D\}$ is relatively compact in $E$. Let $t \in J$ be a fixed and let $0<\varepsilon<t \leq b$. For $z \in D$ we define

$$
F_{\varepsilon}(z)(t)=U(t, t-\varepsilon) \int_{0}^{t-\varepsilon} U(t-\varepsilon, s) f\left(s, z_{\rho\left(s, x_{s}+z_{s}\right)}+x_{\rho\left(s, x_{s}+z_{s}\right)}\right) d s .
$$

Since $U(t, s)$ is a compact operator, and the set $\Lambda_{\varepsilon}:=\left\{\left(F_{\varepsilon} z\right)(t): z \in D\right\}$ is the image of bounded set of $E$ by $U(t, s)$ then $\Lambda_{\varepsilon}$ is precompact in $E$. Furthermore, for $z \in D$, we have

$$
\left|F(z)(t)-F_{\varepsilon}(z)(t)\right| \leq \int_{t-\varepsilon}^{t}\|U(t, s)\|_{B(E)}\left|f\left(s, z_{\rho\left(s, x_{s}+z_{s}\right)}+x_{\rho\left(s, x_{s}+z_{s}\right)}\right)\right| d s
$$




$$
\begin{aligned}
& \leq \int_{t-\varepsilon}^{t}\|U(t, s)\|_{B(E)} p(s)\left(\left\|z_{\rho\left(s, x_{s}+z_{s}\right)}+x_{\rho\left(s, x_{s}+z_{s}\right)}\right\|_{\mathcal{B}}+1\right) d s \\
& \leq \xi \widehat{M} \int_{t-\varepsilon}^{t} e^{-\omega(t-s)} p(s) d s .
\end{aligned}
$$

The right-hand side tends to zero as $\varepsilon \rightarrow 0$, then $F_{\varepsilon}(z)$ converge uniformly to $F(z)$ which implies that $D(t)$ is precompact in $E$.

Finally, it rest to show that $F$ is equiconvergent.

Let $z \in D$, then from the assumptions $\left(H_{1}\right),\left(H_{2}\right)$ and (5) we have

$$
|(F z)(t)| \leq \widehat{M} \xi \int_{0}^{t} e^{-\omega(t-s)} p(s) d s,
$$

it follows immediately by (5) that $|(F z)(t)| \longrightarrow 0$ as $t \rightarrow+\infty$.

Then

$$
\lim _{t \rightarrow+\infty}|(F z)(t)-(F z)(+\infty)|=0
$$

which implies that $F$ is equiconvergent.

\section{Step 2.}

$L$ is a contraction.

Let $z, \bar{z} \in X_{0}$, then by $\left(H_{1}\right),\left(H_{4}\right)-\left(H_{6}\right)$ and (5) and for each $t \in J$ we have

$$
\begin{aligned}
|(L z)(t)-(L \bar{z})(t)| \leq & \left|g\left(t, x_{\rho\left(t, x_{t}+z_{t}\right)}+z_{\rho\left(t, x_{t}+z_{t}\right)}\right)-g\left(t, x_{\rho\left(t, x_{t}+\bar{z}_{t}\right)}+\bar{z}_{\rho\left(t, x_{t}+\bar{z}_{t}\right)}\right)\right| \\
& +\int_{0}^{t}\|U(t, s)\|_{B(E)}\left|A(s) g\left(s, x_{\rho\left(s, x_{s}+z_{s}\right)}+z_{\rho\left(s, x_{s}+z_{s}\right)}\right)-A(s) g\left(s, x_{\rho\left(s, x_{s}+\bar{z}_{s}\right)}+\bar{z}_{\rho\left(s, x_{s}+\bar{z}_{s}\right)}\right)\right| d s \\
\leq & \left\|A^{-1}(t)\right\|_{B(E)}\left|A(t) g\left(t, x_{\rho\left(t, x_{t}+z_{t}\right)}+z_{\rho\left(t, x_{t}+z_{t}\right)}\right)-A(t) g\left(t, x_{\rho\left(t, x_{t}+\bar{z}_{t}\right)}+\bar{z}_{\rho\left(t, x_{t}+\bar{z}_{t}\right)}\right)\right| \\
& +\widehat{M} \int_{0}^{t} e^{-\omega(t-s)}\left|A(s) g\left(s, x_{\rho\left(s, x_{s}+z_{s}\right)}+z_{\rho\left(s, x_{s}+z_{s}\right)}\right)-A(s) g\left(s, x_{\rho\left(s, x_{s}+\bar{z}_{s}\right)}+\bar{z}_{\rho\left(s, x_{s}+\bar{z}_{s}\right)}\right)\right| d s \\
\leq & \ell \widetilde{M}\left\|z_{\rho\left(t, x_{t}+z_{t}\right)}-\bar{z}_{\rho\left(t, x_{t}+\bar{z}_{t}\right)}\right\|_{\mathcal{B}}+\widehat{M} \int_{0}^{t} e^{-\omega(t-s)}\left\|z_{\rho\left(s, x_{s}+z_{s}\right)}-\bar{z}_{\rho\left(s, x_{s}+\bar{z}_{s}\right)}\right\|_{\mathcal{B}} d s .
\end{aligned}
$$

So, by Lemma 3.3 we obtain

$$
\begin{aligned}
|(L z)(t)-(L \bar{z})(t)| & \leq y\left(\ell \widetilde{M}+\widehat{M} \int_{0}^{t} e^{-\omega(t-s)} d s\right)\|z-\bar{z}\|_{x_{0}} \\
& \leq y\left(\ell \widetilde{M}+\frac{\widehat{M}}{\omega}\right)\|z-\bar{z}\|_{x_{0}} .
\end{aligned}
$$

Therefore

$$
\|L z-L \bar{z}\|_{x_{0}} \leq y\left(\ell \widetilde{M}+\frac{\widehat{M}}{\omega}\right)\|z-\bar{z}\|_{x_{0}} .
$$

Thus, the operator $L$ is a contraction. 
In order to apply Theorem 2.4, we must check hypothesis (ii) does not hold, i.e., prove that the set

$$
D_{\lambda}=\left\{z \in X_{0}: z=\lambda L\left(\frac{z}{\lambda}\right)+\lambda F(z) \text { for } \lambda \in(0,1)\right\},
$$

is bounded. Let $z \in D_{\lambda}$ then for each $t \in J$, we have

$$
z(t)=\lambda L\left(\frac{z}{\lambda}\right)(t)+\lambda F(z)(t)
$$

Then we get

$$
\begin{aligned}
|z(t)| \leq & \lambda\|U(t, 0)\|_{B(E)}|| A^{-1}(t)\left\|_{B(E)}|A(t) g(0, \phi)|+\right\| A^{-1}(t) \|_{B(E)}\left|A(t) g\left(t, x_{\rho\left(t, x_{t}+z_{t}\right)}+\frac{z_{\rho\left(t, x_{t}+z_{t}\right)}}{\lambda}\right)\right| \\
& +\lambda \int_{0}^{t}\|U(t, s)\|_{B(E)}\left|A(s) g\left(s, x_{\rho\left(s, x_{s}+z_{s}\right)}+\frac{\left.z_{\rho\left(s, x_{s}+z_{s}\right)}\right)}{\lambda}\right)\right| d s \\
& +\lambda \int_{0}^{t}\|U(t, s)\|_{B(E)}\left|f\left(s, x_{\rho\left(s, x_{s}+z_{s}\right)}+z_{\rho\left(s, x_{s}+z_{s}\right)}\right)\right| d s . \\
\leq & \lambda \widehat{M} \widetilde{M} \zeta(t)\|\phi\|_{\mathcal{B}}+\lambda \widetilde{M} \zeta(t)\left\|x_{\rho\left(t, x_{t}+z_{t}\right)}+\frac{z_{\rho\left(t, x_{t}+z_{t}\right)}}{\lambda}\right\|_{\mathcal{B}}+\lambda \widehat{M} \int_{0}^{t} e^{-\omega(t-s)} \zeta(s)\left\|x_{\rho\left(s, x_{s}+z_{s}\right)}+\frac{z_{\rho\left(s, x_{s}+z_{s}\right)}}{\lambda}\right\|_{\mathcal{B}} d s \\
& +\lambda \widehat{M} \int_{0}^{t} e^{-\omega(t-s)} p(s)\left(\left\|x_{\rho\left(s, x_{s}+z_{s}\right)}+z_{\rho\left(s, x_{s}+z_{s}\right)}\right\|_{\mathcal{B}}+1\right) d s .
\end{aligned}
$$

Thus, by Lemma 3.3 we have

$$
|z(t)| \leq \widehat{M} \widetilde{M} \zeta^{\star}\|\phi\|_{\mathcal{B}}+\widetilde{M} \zeta^{\star} y\left(\|z\|_{x_{0}}+\mu\right)+\frac{\widehat{M} \zeta^{\star}}{\omega}\left(\|z\|_{x_{0}}+\mu\right)+\widehat{M} y\left(\|z\|_{x_{0}}+\mu+1\right)\|p\|_{L^{1}},
$$

with $\mu:=(\widehat{M}+1)\|\phi\|_{\mathcal{B}}$ and $\zeta^{\star}:=\sup _{t \in J}|\zeta(t)|$.

Therefore,

$$
\|z\|_{x_{0}} \leq \frac{\widehat{M} \widetilde{M} \zeta^{*}\|\phi\|_{\mathcal{B}}+\widetilde{M} \zeta^{*} y \mu+\frac{\widehat{M} \zeta^{*}}{\omega} \mu+\widehat{M}(\mu+1)\|p\|_{L^{1}}}{\left(1-\widetilde{M} \zeta^{*} y-\frac{\widehat{M} \zeta^{*}}{\omega}-\widehat{M} y\right)}:=c,
$$

which implies that $D_{\lambda}$ is bounded.

Thus by Theorem 3.4 the operator $T$ has at least one fixed point which is a mild solution of problem (1).

\section{Attractivity of solutions}

In this section we study the local attractivity of solutions the problem (1)

Definition 4.1. [9] We say that solutions of (1) are locally attractive if there exists a closed ball $\bar{B}\left(z^{\star}, \sigma\right)$ in the space $X_{0}$ for some $z^{\star} \in X$ such that for arbitrary solutions $z$ and $\tilde{z}$ of (1) belonging to $\bar{B}\left(z^{\star}, \sigma\right)$ we have

$$
\lim _{t \rightarrow+\infty}(z(t)-\tilde{z}(t))=0
$$

Under the assumptions of Section 3, let $z^{*}$ be a solution of $(1)$ and $\bar{B}\left(z^{*}, \sigma\right)$ the closed ball in $X_{0}$ with $\sigma$ satisfying the following inequality

$$
\sigma \geq \frac{2 \widehat{M}\|p\|_{L^{1}}}{1-\widetilde{M} \ell y-\frac{\widehat{M} \ell y}{\omega}-2 \widehat{M} y\|p\|_{L^{1}}} .
$$


Moreover, we assume that

$$
\lim _{t \rightarrow \infty} \zeta(t)=0 \quad \text { and } \quad \lim _{t \rightarrow \infty} \int_{0}^{t} e^{-\omega(t-s)} \zeta(s) d s=0,
$$

where $\zeta$ is the function in $\left(H_{6}\right)$.

Then, for $z \in \bar{B}\left(z^{\star}, \sigma\right)$ by $\left(H_{1}\right)-\left(H_{2}\right)$ and (5) we have

$$
\begin{aligned}
\left|(T z)(t)-z^{\star}(t)\right|= & \left|(T z)(t)-\left(T z^{\star}\right)(t)\right| \\
\leq & \left\|A^{-1}(t)\right\|_{B(E) \mid}\left|A(t) g\left(t, z_{\rho\left(t, x_{t}+z_{t}\right)}+x_{\rho\left(t, x_{t}+z_{t}\right)}\right)-A(t) g\left(t, z_{\rho\left(t, x_{t}+z_{t}\right)}^{\star}+x_{\rho\left(t, x_{t}+z_{t}\right)}\right)\right| \\
& +\int_{0}^{t}\|U(t, s)\|_{B(E) \mid}\left|f\left(s, z_{\rho\left(s, x_{s}+z_{s}\right)}+x_{\rho\left(s, x_{s}+z_{s}\right)}\right)-f\left(s, z_{\rho\left(s, x_{s}+z_{s}\right)}^{\star}+x_{\rho\left(s, x_{s}+z_{s}\right)}\right)\right| d s \\
& +\int_{0}^{t}\|U(t, s)\|_{B(E) \mid}\left|A(s) g\left(s, z_{\rho\left(s, x_{s}+z_{s}\right)}+x_{\rho\left(s, x_{s}+z_{s}\right)}\right)-A(s) g\left(s, z_{\rho\left(s, x_{s}+z_{s}\right)}^{\star}+x_{\rho\left(s, x_{s}+z_{s}\right)}\right)\right| d s \\
\leq & \widetilde{M} \ell\left\|z_{\rho\left(t, x_{t}+z_{t}\right)}-z_{\rho\left(t, x_{t}+z_{t}\right)}^{\star}\right\|_{\mathcal{B}}+\widehat{M} \ell \int_{0}^{t} e^{-\omega(t-s)}\left\|z_{\rho\left(s, x_{s}+z_{s}\right)}-z_{\rho\left(s, x_{s}+z_{s}\right)}^{\star}\right\|_{\mathcal{B}} d s \\
& +\widehat{M} \int_{0}^{t} e^{-\omega(t-s)} p(t)\left(\left\|z_{\rho\left(s, x_{s}+z_{s}\right)}+x_{\rho\left(s, x_{s}+z_{s}\right)}\right\|_{\mathcal{B}}+\left\|z_{\rho\left(s, x_{s}+z_{s}\right)}^{\star}+x_{\rho\left(s, x_{s}+z_{s}\right)}\right\|_{\mathcal{B}}+2\right) d s \\
\leq & \widetilde{M} \ell y \rho+\frac{\widehat{M} \ell y \rho}{\omega}+2 \widehat{M}(y \rho+1)\|p\|_{L^{1}} \\
\leq & \widetilde{M} \ell y \rho+\frac{\widehat{M} \ell y \rho}{\omega}+2 \widehat{M}(y \rho+1)\|p\|_{L^{1}} \\
\leq & \sigma .
\end{aligned}
$$

Therefore, we get $T\left(\bar{B}\left(z^{\star}, \sigma\right)\right) \subset \bar{B}\left(z^{\star}, \sigma\right)$.

So, for each $z \in \bar{B}\left(z^{\star}, \rho\right)$ solution of problem (1) and $t \in J$, we have

$$
\begin{aligned}
\left|z(t)-z^{*}(t)\right|= & \left|(T z)(t)-\left(T z^{\star}\right)(t)\right| \\
\leq & || A^{-1}(t) \|_{B(E)}\left|A(t) g\left(t, z_{\rho\left(t, x_{t}+z_{t}\right)}+x_{\rho\left(t, x_{t}+z_{t}\right)}\right)-A(t) g\left(t, z_{\rho\left(t, x_{t}+z_{t}\right)}^{\star}+x_{\rho\left(t, x_{t}+z_{t}\right)}\right)\right| \\
& +\int_{0}^{t}\|U(t, s)\|_{B(E)}\left|f\left(s, z_{\rho\left(s, x_{s}+z_{s}\right)}+x_{\rho\left(s, x_{s}+z_{s}\right)}\right)-f\left(s, z_{\rho\left(s, x_{s}+z_{s}\right)}^{\star}+x_{\rho\left(s, x_{s}+z_{s}\right)}\right)\right| d s \\
& +\int_{0}^{t}\|U(t, s)\|_{B(E)}\left|A(s) g\left(s, z_{\rho\left(s, x_{s}+z_{s}\right)}+x_{\rho\left(s, x_{s}+z_{s}\right)}\right)-A(s) g\left(s, z_{\rho\left(s, x_{s}+z_{s}\right)}^{\star}+x_{\rho\left(s, x_{s}+z_{s}\right)}\right)\right| d s \\
\leq & \widetilde{M} \zeta(t)\left(\left\|z_{\rho\left(t, x_{t}+z_{t}\right)}+x_{\rho\left(t, x_{t}+z_{t}\right)}\right\|_{\mathcal{B}}+\left\|z_{\rho\left(t, x_{t}+z_{t}\right)}^{\star}+x_{\rho\left(t, x_{t}+z_{t}\right)}\right\|_{\mathcal{B}}\right) \\
& +\widehat{M} \int_{0}^{t} e^{-\omega(t-s)} p(s)\left(\psi\left(\left\|z_{\rho\left(s, x_{s}+z_{s}\right)}+x_{\rho\left(s, x_{s}+z_{s}\right)}\right\|_{\mathcal{B}}\right)+\psi\left(\left\|z_{\rho\left(s, x_{s}+z_{s}\right)}^{\star}+x_{\rho\left(s, x_{s}+z_{s}\right)}\right\|_{\mathcal{B}}\right)\right) d s \\
& \left.+\widehat{M} \int_{0}^{t} e^{-\omega(t-s)} \zeta(s)\left(\left\|z_{\rho\left(s, x_{s}+z_{s}\right)}+x_{\rho\left(s, x_{s}+z_{s}\right)}\right\|_{\mathcal{B}}+\| z_{\rho\left(s, x_{s}+z_{s}\right)}^{\star}+x_{\rho\left(s, x_{s}+z_{s}\right)}\right) \|_{\mathcal{B}}\right) d s \\
& 2 y \widetilde{M}(\sigma+\mu) \zeta(t)+2 \widehat{M} \psi(y \sigma+y \mu) \int_{0}^{t} e_{0}^{-\omega(t-s)} p(s) d s+2 y \widehat{M}(\sigma+\mu) e_{0}^{-\omega(t-s)} \zeta(s) d s \\
\leq &
\end{aligned}
$$


Hence, from $\left(H_{3}\right)$ and (6), we conclude that

$$
\lim _{t \rightarrow \infty}|z(t)-\tilde{z}(t)|=0 .
$$

Consequently, the solutions of problem (1) are locally attractive.

\section{An example}

To apply the results of sections 2 and 3 , we consider the problem

$$
\begin{aligned}
& \frac{\partial}{\partial t}\left(v(t, \xi)+\int_{-\infty}^{t} a(t-s) v\left(s-\rho_{1}(t) \rho_{2}(|v(t, \xi)|), \xi\right) d s\right)= \\
& \frac{\partial^{2}}{\partial \xi^{2}} v(t, \xi)+b(t, \xi) v(t, \xi)+\int_{-\infty}^{t} c(s-t) v\left(t-\rho_{1}(t) \rho(|v(t, \xi)|), \xi\right) d s, \\
& \left\{\begin{array}{l}
v(t, 0)=v(t, \pi)=0 \quad t \geq 0, \\
v(s, \xi)=v_{0}(s, \xi), \quad s \in(-\infty, 0], \xi \in[0, \pi],
\end{array}\right.
\end{aligned}
$$

where $\rho_{1}, \rho_{2}: J \rightarrow \mathbb{R}_{+}$and $a, c: \mathbb{R} \rightarrow \mathbb{R}$ are continuous functions, and $b(t, \xi)$ is continuous with respect to $t$.

Let $X=L^{2}([0, \pi])$ and $A(t): D(A) \subset X \rightarrow X$ be the operator defined as follows

$$
A(t) x(\xi)=A x(\xi)+b(t, \xi) x(\xi)
$$

where $A x=x^{\prime \prime}$ be the operator with domain $D(A)=\left\{x \in X: x^{\prime \prime} \in X, x(0)=x(\pi)=0\right\}$ which generates an analytic semigroup on $X$.

Now, we note $\mathcal{B}=C_{0} \times L^{p}(h, X)$ the phase space introduced in Hino et al. [18], where $h:(-\infty, 0] \rightarrow \mathbb{R}$ be non-negative measurable function which satisfies the appropriate conditions cited in [18].

For $t \in J, \xi \in[0, \pi]$, and by defining the functions $f, g: J \times \mathcal{B} \rightarrow X$ by

$$
\begin{gathered}
y(t)(\xi)=v(t, \xi), \\
\phi(\tau)(\xi)=v_{0}(\tau, \xi), \text { for }-\infty<\tau \leq 0, \\
g\left(t, y_{t}\right)(\xi)=\int_{-\infty}^{t} a(t-s) y\left(s-\rho\left(t, y_{t}\right)\right)(\xi) d s,
\end{gathered}
$$

and

$$
f\left(t, y_{t}\right)(\xi)=\int_{-\infty}^{t} c(t-s) y\left(s-\rho\left(t, y_{t}\right)\right)(\xi) d s,
$$

where $\rho: J \times \mathcal{B} \rightarrow \mathbb{R}$ is defined by

$$
\rho\left(t, y_{t}\right):=\rho_{1}(t) \rho_{2}(|y(t)(\xi)|),
$$

We can transform the problem (7) - (8) into the abstract form (1).

Thus, under appropriate conditions on the functions $a, b$, and $c$, problem (7)-(8) has a solution which is locally attractive.

Acknowledgement: The authors are grateful to the referees for the careful reading of the paper and for their remarks. 


\section{References}

[1] N.U. Ahmed, Semigroup Theory with Applications to Systems and Control, Pitman Research Notes in Mathematics Series, 246. Longman Scientific \& Technical, Harlow John Wiley \& Sons, Inc. New York, 1991.

[2] W.G. Aiello, H.I. Freedman, J. Wu, Analysis of a model representing stage-structured population growth with statedependent time delay. SIAM J. Appl. Math. 52 (3) (1992), 855-869.

[3] S. Baghli and M. Benchohra, Perturbed functional and neutral functional evolution equations with infinite delay in Fréchet spaces, Electron. J. Differential Equations 2008 (69) (2008), 1-19.

[4] S. Baghli and M. Benchohra, Global uniqueness results for partial functional and neutral functional evolution equations with infinite delay, Differential Integral Equations 23 (1\&2) (2010), 31-50.

[5] S. Baghli and $M$. Benchohra, Existence results for semilinear neutral functional differential equations involving evolution operators in Fréchet spaces, Georgian Math. J. 17 (2010), 1072-9176.

[6] A. Caicedo, C. Cuevas, G. M. Mophou, and G. M. N'Guérékata, Asymptotic behavior of solutions of some semilinear functional differential and integro-differential equations with infinite delay in Banach spaces. J. Franklin Inst. 349 (2012), 1-24.

[7] T. A. Burton and C. Kirk, A fixed point theorem of Krasnoselskii type, Math. Nachrichten 189 (1998), 23-31.

[8] C. Corduneanu, Integral Equations and Stability of Feedback Systems, Acadimic Press, New York, 1973.

[9] B.C. Dhage, V. Lakshmikantham, On global existence and attractivity results for nonlinear functional integral equations, Nonlinear Anal. 72 (2010), 2219-2227.

[10] J. P.C. dos Santos, On state-dependent delay partial neutral functional integro-differential equations, Appl. Math. Comput. 216 (2010) 1637-1644.

[11] K. J. Engel and R. Nagel, One-Parameter Semigroups for Linear Evolution Equations, Springer-Verlag, New York, 2000.

[12] X. Fu, Existence and stability of solutions to neutral equations with infinite delay, Electron. J. Differential Equations, Vol. 2013 (2013), No. 55, pp. 1-19.

[13] J. K. Hale, Theory of Functional Differential Equations, Springer-Verlag, New York, 1977.

[14] J. Hale and J. Kato, Phase space for retarded equations with infinite delay, Funkcial. Ekvac. 21 (1978), 11-41.

[15] J. K. Hale and S. M. Verduyn Lunel, Introduction to Functional Differential Equation, Applied Mathematical Sciences 99 , Springer-Verlag, New York, 1993.

[16] E. Hernández M. and M. A. McKibben, On state-dependent delay partial neutral functional-differential equations, Appl. Math. Comput. 186 (2007), 294-301.

[17] E. Hernández, M. A. McKibben and H. R. Henríquez, Existence results for partial neutral functional differential equations with state-dependent delay Math. Comput. Modelling 49 (2009), 1260-1267.

[18] Y. Hino, S. Murakami, and T. Naito, Functional Differential Equations with Unbounded Delay, Springer-Verlag, Berlin, 1991.

[19] V. Kolmanovskii, and A. Myshkis, Introduction to the Theory and Application of Functional-Differential Equations. Kluwer Academic Publishers, Dordrecht, 1999.

[20] V. Lakshmikantham, L. Wen and B. Zhang, Theory of Differential Equations with Unbounded Delay, Kluwer Acad. Publ., Dordrecht, 1994.

[21] A. Pazy, Semigroups of Linear Operators and Applications to Partial Differential Equations, Springer-Verlag, New York, 1983.

[22] N. Van Minh, Gaston M. N'Guérékata, and C. Preda. On the asymptotic behavior of the solutions of semilinear nonautonomous equations. Semigroup Forum 87 (2013), 18-34.

[23] J. Wu, Theory and Application of Partial Functional Differential Equations, Springer-Verlag, New York, 1996. 